\title{
OPINOIN \\ FALSIFIABILITY OF THEORIES IN THE BIOLOGICAL SCIENCES
}

\author{
M.C.M. Iqbal \\ Institute of Fundamental Studies, Hantana Road, Kandy, Sri Lanka. \\ Accepted 5 December 2007
}

\begin{abstract}
Biology is generally accepted as a mainstream scientific discipline. However, philosophers of science have questioned the scientific method applied in biological sciences, specifically in evolutionary biology, ever since Karl Popper formulated his principle of falsification. Thus the only major theory in biology, Darwin's theory of evolution, was referred to by Popper as a metaphysical programme. He contended that the theory of evolution is a tautology and laws (if any) in the biological sciences should be unrestricted and universal. As biologists since then have pointed out, biology is a unique science, which requires unique methods to explain its phenomena. The principle of falsification and its application to biological sciences, the uniqueness of biology as a science necessitating different and equally valid scientific methods are discussed.
\end{abstract}

\section{INTRODUCTION}

The scientific revolution began in the $17^{\text {th }}$ century with the method of induction, introduced by Francis Bacon (1561 - 1626), to seek explanation for natural phenomena. This is a method of measuring and recording observations without a preconceived hypothesis. The earliest sciences associated with the scientific revolution were astronomy and mechanics, based on observation and mathematics. This led to the formulation of the laws of gravitation by Isaac Newton and his fundamental contributions in mechanics, optics and mathematics. During this time the oldest scientific society, The Royal Society, was formed in London, whose objective was to replace philosophy as the source of knowledge, by the scientific method for seeking explanations. With the rise of science, philosophy changed its emphasis from seeking knowledge to determining the methods of seeking knowledge and distinguishing between rational sources of knowledge (science) and nonrational sources (spiritualism, astrology etc.). The development of philosophy is punctuated by the rise and fall of different schools of thought to keep apart science and non-science.

To distinguish science from the nonsciences, philosophical definitions of science were proposed in the $19^{\text {th }}$ century and early $20^{\text {th }}$ century. The most significant of these was the principle of falsification by Karl Popper (1934).
This states that a scientific statement is liable to be proved wrong (falsified), can be tested by observation and experiment, and successfully makes predictions. This effectively kept out a host of pseudo-sciences such as astrology, spiritualism, phrenology, Freudianism, Marxism etc. from the sciences which was essentially physics at that time. Popper also extended his arguments to Darwin's Theory of Evolution stating that it is not falsifiable and hence belongs to metaphysics. A metaphysical theory, according to Popper, cannot be verified or falsified.

This paper looks at the philosophical controversies and contradictions in the biological sciences and the theory of evolution, and the evidence from biology contradicting Popper's views. It is an attempt to underline the uniqueness of biology as a science, and its contrast with the physical sciences and why it does not and cannot subscribe to Popperian philosophy and his scientific method of falsification, testing and prediction. It is also an attempt to synthesize the views of biologists and philosophers towards this end.

\section{Popper's principle of Falsification}

Popper proposed his principle of falsification to keep out the pseudo-sciences, he being very critical of Freudianism and Marxism. Although a rationalist himself and a supporter of Darwin's theory, he was unequivocal on what is science 
and that Darwinism does not offer a causal explanation for adaptive evolution shown by any single organ or organism. He wrote in his book “Objective Knowledge” (1972), 'Darwin’s discovery of the theory of natural selection has often been compared to Newton's discovery of the theory of gravitation. This is a mistake. Newton formulated a set of universal laws intended to describe the interaction, and consequent behaviour, of the physical universe. Darwin's theory of evolution proposed no such universal laws. There are no Darwinian laws of evolution'. He further writes, 'Nevertheless, Darwin's revolutionary influence upon our picture of the world around us was at least as great, though not as deep, as Newton's. For Darwin's theory of natural selection showed that it is in principle possible to reduce teleology to causation by explaining, in purely physical terms, the existence of design and purpose in the world'.

The concept of universal laws was paramount to fit into Popper's concept of science. His primary purpose was to isolate science from the pseudosciences (or metaphysics), which at that time was distinguished by the empirical method, an inductive process, proceeding from observation and experiment (Popper 1963).

Popper's attention to the philosophy of science began in 1919, when radical theories were turning around the sciences and social order. Besides Einstein's theory of relativity, he was interested in Marx's theory of history, Freud's psychoanalysis and Adler's individual psychology. Being dissatisfied with the latter three theories and their claim to scientific status he proposed his principle of falsification to keep apart the sciences from the non-sciences. He stated that his sole purpose of proposing the criterion of falsification was to draw a line between the empirical sciences and all other statements, whether they are religious, metaphysical or pseudo-scientific (Popper 1963). Writing in "The logic of scientific discovery" Popper (1934) states that science makes different types of statements. He identifies these statements as follows. Singular statements refer to only certain finite regions of space and time e.g. 'Here is a black crow' or an occurrence 'A glass of water has just being upset here'. In contrast to singular statements are universal statements. Popper distinguishes between strictly universal statements and numerically universal statements. Strictly universal statements refer to a class of objects which are spatiotemporally unrestricted, being true for any place and any time. An example is Newton's laws of gravitation and Einstein's theory of relativity. Numerically universal statements refer to a finite class of objects, which are spatiotemporally restricted. Thus Popper's concept of a law of nature can only be a strictly universal statement.

Popper's views on his principle of falsification have come in for criticism. Popper relentlessly argued against the method of induction in science - science does not progress by confirming a hypothesis but only by falsifying it. Scientists should look for disproving their conjectures than looking for instances for confirming it. Gardner (2001), contends that falsification is much rarer in the sciences than searches for confirming instances and it could also be based on a faulty observation. Scientists in general conduct experiments to confirm a conjecture and not to prove it wrong. Astronomers look for signs of water on Mars; they do not think they are making efforts to falsify the conjecture that Mars never had water. Gardner is of the opinion that induction is firmly embedded in the way philosophers of science and even ordinary people talk and think. Popper's views were also at odds with The Vienna Circle. Popper did not accept the verification of a hypothesis by experimental observation as a confirmation of the hypothesis. For Popper, a universal statement could be falsified but not verified. He could not make sense of the idea that a scientific theory becomes more probable when a prediction is verified. The Vienna Circle and associated philosophers did not agree with this asymmetric treatment of verification and falsification (Corfield et al. 2005). Though Popper attempts to distinguish between science and non-science, those disciplines that are based on historical evidence (cosmology, geology, evolutionary biology) also ended up as metaphysics.

\section{Biology as a Science}

Biology as a science developed much after physics and chemistry. Unlike physics and chemistry whose laws have no spatial or temporal restriction, biology is limited to the earth and to a time frame beginning from the inception of life to the present. However, in spite of these restrictions, the principles governing life are universal to wherever and whenever life forms occur. Thus, 'universal' is a domain determined by the entities, it is a function of. Biology and is considered to be composed of physics and chemistry. However, many attributes of life cannot be reduced to the known 
laws of physics and chemistry. While the structure of the gene can be reduced to the molecules of chemistry and the double helix structure of the DNA molecule obeys the laws of physics, the expression and functioning of the gene is not governed by physics and chemistry. Biology as a science was a late entrant to the sciences, primarily because its fundamental attributes are determined by physics and chemistry and the vast diversity of life forms had to be brought under a single theory as achieved by Darwin and Mendel and so many others since.

In the physical sciences it is possible for there to be a process of reduction from one level of science to the next level leading ultimately to sub-atomic particles. Biology, in theory can be reduced through biochemistry and chemistry to physics. But life forms, however, simple, do not lend themselves to this reduction. Each level in a biological system is composed of many factors interacting between themselves and at different levels. In genetics the phenotype expression, for example, cannot be read off from the genetic code. Between the phenotype and the gene are populations, Mendelian and molecular genetics, with each level consisting of many interacting entities.

Mayr (1976) considered biology as two largely separate fields that differ in their method, formulation and basic concepts. He identifies them as functional biology and evolutionary biology, which of course overlap into each other. Functional biology is concerned with the functioning of cells and their components, tissues, organs and individual organisms. It attempts to understand the functioning through experiments with controls and manipulating variables, as in physics and chemistry. Evolutionary biology is concerned with all life forms to determine their diversity and the forces that bring about changes in the fauna and flora and their adaptations. The evolutionary biologist is concerned with the historical development leading to changes and the cause of these changes. The experimental method is thus not applicable to explain such events or processes.

Biological processes are simultaneously determined by many factors. Consequently theories in biology are probabilistic and cannot be attributed to a single factor, compared to the physical sciences where causes are identifiable (except at the quantum level, where Heisenberg's Uncertainty Principle shows discrete causes are not always identifiable, but probabilistic). Causation is also complicated in biology. The final effect or outcome is the end result of a chain of events. A phenomenon or process in living organisms can be attributed to two separate causes: a proximate or functional cause and an ultimate or evolutionary cause (Mayr,1998). Proximate causes determine the physiological, developmental and behavioural processes that are controlled genetically. Ultimate causes determine the changes that occur during the evolutionary processes. They lead to changes in the genotype at the population level. For example plant and animal breeding lead to changes of not only individual genes but also gene frequencies in a variety or breed. Such changes are not accessible to physical or chemical laws. Thus proximate causes answer the question "How did it happen?" while ultimate causes explain "Why did it happen?" (Mayr, 1998). Thus Popper's principles are applicable to proximate causes that ask the question "How?", but not to ultimate causes in biology.

Writing in his book "Objective Knowledge" (1972), Popper contends that neither Darwin, nor any Darwinian has so far given an actual causal explanation of the adaptive evolution of any single organism or any single gene. He further questions 'Why should natural selection have produced anything beyond the general increase in rates of reproduction, and the elimination of all but the most fertile breeds?' Popper's understanding of biology and evolutionary theory did not permit him to comprehend the nature of natural selection and that it went beyond simply increasing the rates of reproduction and he was ignorant of the role of adaptive evolution. The theory of evolution brings together a wide spectrum of evidence to support itself: adaptive radiation, speciation, camouflaging (see below, Is 'the principle of Natural Selection' a tautology?), succession of fossil forms in geology, biogeography of species distribution, homologous structures, vestigial organs, character reversions amongst others. Darwin's monumental contribution to biology in his book "On the Origin of Species" (1859), is the unification of all living organisms under a single theory able to explain their diversity, inter-relations and evolution.

As Mayr argues, biology is like physics and chemistry a science. But, biology is not a science like physics and chemistry; it is rather an autonomous science on par with the equally autonomous physical sciences. 


\section{Historical explanations: an alternative to the scientific method}

The scientific method involves experimentation either in the field or laboratory, where experiments are designed, conducted, repeated, variables restricted, new variables introduced and conclusions drawn. In short, the experimental material is controlled and manipulated. This method is inappropriate to explain some of the unique phenomena in biology, geology and astronomy whose events have occurred in the past and need to be reliably reconstructed and tested. The method used here is constructing a historical narrative (or explanation), which differs from conventional experiments. To explain unique events in biology Mayr (1998) explains how a 'historical narrative' is constructed. It is the study of all known facts relating to a particular problem, inferring the possible consequences from the reconstructed constellation of factors and attempt to construct a scenario that would explain the observed facts. This is how Darwin proposed his theory of evolution. Gould (1989) identifies the fundamental differences between conventional science and historical explanations. Verification by repetition does not arise in historical explanations, since the uniqueness of detail would not occur again due to the laws of probability and time's irreversibility. The complex events of the past are not reduced to simple events, but looked at as a whole. Prediction, important in the empirical method, is not an issue since the events are a result of many factors coming together at a particular time.

Gould (1989) states that sciences of history (cosmology, geology, evolutionary biology) use a different method of explanation based on comparative and observational richness of data, capable of achieving equally firm conclusions as by the conventional scientific method. It was the lack of empiricism that led Popper to label the theory of evolution as metaphysics. Whatever the method, it should be verifiable or testable independently to judge the hypothesis. Gould states that only the theory of evolution is able to coordinate the disparate data of embryology, bio-geography, the fossil record, vestigial organs, taxonomic relations etc. The theory of evolution explains the diversity of species and their similarities and differences. It also accounts for the fossil records, where the deposition of fossils at different points of time in the past and their dating, indicates a succession of organisms over time. The fossil records suggest, according to the theory of evolution, that organisms evolved in a hierarchy of stages and the mechanism for this change was natural and other forms of selection. The theory of evolution is not only consistent with the observations made by him, but also with the independent sources of data now available. The mechanism of inheritance, on which evolution and natural selection operates, has remained consistent from Mendelian genetics, through the discovery of chromosomes, to the biochemical nature of the gene. Caplan (1981) describes in a nutshell what is theory and fact about evolution: the theory of evolution is a true theory as any other in science. However, it is not a fact. Evolution is a fact, and the theory of evolution tries to explain this fact.

Although a historical narrative cannot be proven 'true', it is yet open to falsification and can be tested again and again. Dawkins (1986) advances a simple test to counter the argument that the theory of evolution is an unfalsifiable tautology: 'If a single, well-verified mammal skull were to turn up in 500 million year old rocks, our whole modern theory of evolution would be utterly destroyed'. According to Mayr (1998), an important objective of a historical narrative is to discover causal factors that contributed to later events in a historical sequence. Historical narrative does not abandon causality (which is important in the physical sciences), but it is a causality arrived at strictly empirically, not related to any law, but rather explains a unique case.

\section{Is 'the principle of Natural Selection' a tautology?}

The theory of evolution explains evolution based on the principles of natural selection. Natural selection is associated with the phrase 'survival of the fittest'. Although popularly associated with Darwin, it was formulated by an English philosopher, Herbert Spencer (1820 1903) and apparently disliked by Darwin. Some critics of the theory of evolution have taken a closer look at this phrase. Their criticism is 'survival' means staying alive; but what about the 'fittest'? That too, in the end, only seems to mean those that survive, which is a circular statement - a tautology (Ridley 1985). Popper remarked that 'there is hardly any possibility of testing a theory as feeble as this'. Is this criticism valid? Ridley (1985) argues that it is not. He considers the well known example of the development of camouflage by the moth Biston betularia in industrial regions of Great Britain. The moth occurs in forms; a dark melanic form and a lighter peppered form. Before the industrial revolution the peppered type was more common. As industrial activity increased, the 
melanic type increased in frequency in industrial areas, while the peppered type remained common in non-industrial areas. As industrial activity decreased in some industrial areas, the peppered type was more common. The explanation for these phenomena was that the soot from the factories kills the lichens on the tree trunks, leaving the trees bare and dark. The peppered type is camouflaged on a lichencovered trunk, but not on bare lichen-less trunk. The melanic type is camouflaged on bare lichenless trunk, but stands out on the lichen-covered trunk.

The change in relative frequencies of the two forms of moths was caused by bird predation against a changing background. This example shows natural selection at work. The type that survived better, in one area, increased in frequency. There was an evolutionary change, towards the better adapted form, in the moth population. Thus the form that survived and reproduced successfully increased in frequency, which is a tautology. But, why does one form survive better than the other? Ridley explains that the mechanism of adaptation is camouflage. Those moths that were camouflaged and so escaped the visually hunting birds, survived. Camouflage was the mechanism of adaptation. Camouflage was possible, because it was an inherited trait and the reshuffling of genes during reproduction produced a range of wing colours on which natural selection was able to act. This is the causal explanation of adaptive evolution that Popper and other critics did not find.

The principle of natural selection was studied in detail by Campbell and Roberts (2005). They consider past alternative explanations offered by evolutionary biologists as to why the principle of natural selection is not a tautology and offered their own model of evolution by natural selection, which they claim is testable. Their model implies that better adaptation by organisms to their environment, is the cause of differential reproduction, leading to natural selection. Fitness is more than just survival. It is ultimately manifested in better reproduction that leaves offsprings for the next generation.

\section{Testing and Prediction in Biology}

Testing a hypothesis and making predictions from a theory are fundamental prerequisites to accepting a theory in science, as set out by Popper (1934). A good theory enables a correct prediction under a particular set of factors (this prediction is unlike chronological prediction, which is foretelling the future). Falsification is not suited to test probabilistic theories, which include most theories in biology and evolutionary biology where historical narratives are constructed to explain certain observations where it is difficult if not impossible to falsify an invalid theory (Mayr, 1998). Biological regularity rarely has the universality of physical laws. Whereas in functional biology theories have high predictive value (eg. aerobic life cannot survive in the absence of oxygen), it is impossible to predict the course of evolution or extinction of a species in evolutionary biology, which is controlled by a complex set of factors (Mayr, 1998). He continues to state that predictions in biology and, for that matter, quantum physics are at best probabilistic and it is more important for the biologist that the theory is useful in solving problems than to survive the test of prediction.

In the sciences where experimental verification is not possible, as in evolution, geology and astronomy, theories are tested by additional observations and on alternative evidence. For example in evolutionary theory, plant and animal taxa are assigned to specific geological periods. Thus, man originated in the Cenozoic era and if human fossils were found in the Mesozoic, it would contradict the theory of common descent by Darwin. Another way of testing a theory in biology is to use an entirely different set of facts (Mayr, 1998). A phylogenetic tree, for a group of organisms, can be constructed from morphological evidence and independently from molecular evidence, which enables to test the degree of congruency between the two trees. An example is the search for 'African Eve' from palaeontological and molecular evidence.

Stamos (1996) in a detailed review of Popper's test for genuine science suggests a reinterpretation of the falsification criteria to accommodate the biological sciences. Popper's sole criterion for a statement to be empirical is that it must be falsifiable. Verifiability is not a criterion. For Popper, strictly existential statements need not be empirical. Such statements are spatio-temporally unrestricted. Consider the statement "there are white ravens (or crows)”. According to Popper (1934), this statement is not empirical but metaphysical because it cannot be falsified. One cannot search the universe to establish there are no white ravens, never was and never would exist. However, the statement can be verified e.g. if a white crow is found. But Popper insists it is not empirical, because the class of potential falsifiers 
is empty. Stamos (1996) states that the concept of what is empirical should also be expanded to verifiable statements, besides falsifiable statements. Thus, strictly existential statements, though verifiable but not falsifiable, would nevertheless be empirical and hence belong to science. He includes as empirical, not only universal statements but also singular statements including historical statements as found in evolutionary biology (eg. Homo sapiens had lived approximately 200,000 years ago from Homo erectus, which in turn had appeared approximately 1,500,000 years ago from Homo habilis).

\section{Laws in Biology}

Laws established in the physical sciences are universal (not restricted spatio-temporally) and applicable without exception with invariant probabilities. Philosophers have argued on the status of empirical laws in the physical and biological sciences. Some believe this dichotomy is justified, others argue that there are strict or universal laws and laws with conditions (ceteris paribus laws). Some also argue that the concept of strict universal laws should be modified to accommodate non-empirical laws. These wide ranging issues are referred to by Elgin (2006), and are beyond the scope of this article. The arguments for the absence of universal laws in biology are that organisms are too complex and behave differently even within the same species under the same circumstances and objects of biological generalization - genes, organisms, species - are constantly changing being subject to the forces of evolution (Elgin, 2006). With the accumulation of scientific evidence from diverse fields, biologists have questioned this dogma and looked for underlying principles and design beneath the complexity in biology. Far ahead of his time, Thompson in 1917, attempted to bring a unity of purpose over the diversity spawned by biology, by reducing biological phenomena to mathematical principles in his classic book "On Growth and Form”. Alon (2007) effectively describes the complexity in biology defying comprehension: 'Complex is perhaps the most common adjective used to describe biological phenomena. In every cell, complex networks of interactions occur between thousands of metabolites, proteins and DNA. Every interaction is itself a complex dance between exquisitely shaped proteins, designed to interface with each other if conditions are right. And every protein looks like tangled strands of spaghetti festooned with atomic appendages. So where is the simplicity?' Alon goes on to suggest that the place to look for is in the biological networks of interaction. The structure of networks that regulate genes seems to be built from only a few types of patterns called network motifs. These motifs appear again and again having the same pattern of interaction but with different genes. The small set of network motifs discovered in bacteria also regulates genes in plants and animals. Alon argues that this simplicity in biological systems can be further extended by considering gene action using mathematical models, instead of the details of synthesis and working of proteins. Such models would include whether $\mathrm{X}$ activates or $\mathrm{Y}$ inhibits the different components in a particular gene action. Alon emphasizes that simplicity in biology is necessary to discover general principles to decipher apparently incomprehensible biological networks to eventually make sense of biology on the level of an entire cell, tissue or organism.

An underlying feature in biological organisms is the quarter power law. In animals, from mice to elephants, the body mass and metabolic rates are related by an allometric equation, where the exponent is a multiple of $1 / 4$. In 1932, Max Kleiber, an animal scientist, determined from measurements of body size and metabolic rate that the exponential relationship was 0.74 (ie. nearly $3 / 4$ ), for a broad spectrum of animals (cited by West, 1999). The elephant with a $10^{4}$ times the mass of a chicken, has a metabolic rate of one-tenth that of the chicken. Thus smaller animals (eg. mouse) have a higher metabolic rate and die young while larger animals (eg. whales, elephants) have a slower metabolic rate and live longer. Other relationships include $1 / 4$ for lifespan, $3 / 4$ for age at first reproduction and $1 / 4$ powers of mass for duration of embryonic development (Williams, 1997). The common factor, $1 / 4$, applies to organisms from microbes to higher plants and animals. The ecologists, Enquist and Brown collaborated with the physicist, West to transcend the boundaries of biology and physics to propose that the $1 / 4$ power law derives from physical constraints of an ideal system for distributing nutrients through blood vessels in animals and vascular network in plants (West et al., 1997, 1999, Mackenzie, 1999).

An obvious consequence of evolution is diversity of body sizes ranging over 21 orders of magnitude from $10^{-13} \mathrm{~g}$ for microbes to $10^{8} \mathrm{~g}$ for whales. Within this range of body sizes, evolution has maximized metabolic capacity by increasing the surface area where resources are exchanged with the environment and maximized 
internal efficiency by reducing the distances over which materials are transported and hence the time required for transport (West et al., 1999). The relationship between plant size and number in a given area is obvious. Enquist et al. (1998) showed empirically that total plant resource use scales both within and amongst species as $3 / 4$ power of their body mass. This is the same exponent as for animal metabolic requirement (Damuth, 1981). Enquist, Brown and West developed a model which is a combination of the dynamics of energy transport and the mathematics of fractal geometry that confirms well with observations of living systems and the one-fourth power scaling. The model predicts the scaling exponents measured for many structural and functional variables of mammalian and plant vascular systems. These variables scale with the $1 / 4$ powers of body mass, e.g. $3 / 4$ for metabolic rate, $3 / 4$ for population density, and $1 / 4$ for lifespan (Enquist et al., 1998). For such a common scaling across plants and animal species and structural and functional variables, there should be a unity in the underlying physical properties of the organisms. This was shown to be the distribution of resources (internal transport) and energy metabolism, which is common to all life forms, by these authors. Elgin (2006) argues that these findings are laws in the biological sciences: they are universal in that they assert that any organisms that have the properties in question would exhibit the same behaviour.

It is evident from the foregoing that the physical and biological sciences are not unified with regard to establishment of theories, laws and testing of empirical facts. The philosophies of science are diversified, depending on their school of thought, in their search to determining the goodness of a theory. Biology is a unique science and the classical scientific method is not applicable to historical events. To classify all theories of natural phenomena as either falsifiable or metaphysical would destroy the essence of science: to seek knowledge. Cosmology, geology and evolutionary biology have proved capable by their method of historical reasoning to provide us with a verifiable and better perspective of the living and non-living world, than possible with the falsifiable sciences alone.

Darwin's theory has undergone many changes and modifications, in accordance with new knowledge and interpretations. While the challenges would continue, a philosophy of biology is developing, with contributions from the philosophers of science and scientists in biology. Why do scientists need philosophers to tell them how to do science? It is to underline the methodology in the biological sciences, and thereby secure the validity of their methodology. Mayr (1998) emphasized the role of philosophy in the sciences thus: 'Nevertheless, it must be acknowledged that our sense organs are fallible and our reasoning more so. It is a legitimate task of philosophy to scrutinize the methods by which scientists obtain knowledge, indeed to advise scientists as to the most reliable way to formulate and test theories'.

\section{GLOSSARY OF TERMS USED}

Empiricism - in the philosophy of science, it is a theory of knowledge which emphasizes knowledge derived from experience, especially through deliberate experiments. A central concept in science is that all evidence must be empirical or empirically based, that is dependent on evidence observable by the senses. In a broad sense it is synonymous with experimental.

Metaphysics - is concerned with explaining the nature of reality, beings and the world. It is a philosophical enquiry into subjects beyond the physical world and outside science. It deals with issues of what is beyond experience (ie. it is not empirical).

Tautology - A circular definition eg. natural selection is the survival of the fittest. The fittest are those that survive. Therefore, evolution by natural selection is a tautology.

Induction - The process of deriving general principles from particular facts or instances. Induction was the accepted scientific method after the scientific revolution in the $17^{\text {th }}$ century, introduced by Sir Francis Bacon. The scientist does not have a preconceived hypothesis, and theories are developed by recording and describing observations.

Falsifiability - It is the possibility that a statement can be shown to be false by an observation or experiment. Falsification of a theory leads to a new theory, which has greater explanatory powers. If a theory fails a test to determine its validity, it is said to be falsified. According to Popper, falsification was the only means of eliminating an invalid theory.

Verification and falsification - The statement, "There are white crows" is an existential (or metaphysical) statement. One could look around 
for white crows and if one is found the statement is verified. However, the statement cannot be falsified (one cannot search the universe, in the past, present and future) and hence in Popper's view is not empirical. Newton's theory based on apple falling from a tree is empirical. It is falsified if apples do not fall down.

Historical narrative - Is the construction of a chain of events offering a tentative explanation to a historical phenomenon. The historical narrative is tested with the available facts to best explain the particular event. This is the only method available to explain phenomena in evolutionary biology, geology and cosmology, where conventional scientific methods cannot be applied.

\section{ACKNOWLEDGEMENTS}

I thank the anonymous reviewers for their valuable comments towards improving this manuscript.

\section{REFERENCES}

Alon, U. (2007). Simplicity in Biology. Nature, 446: 497.

Campbell, R. and Robert, J.S. (2005). The structure of evolution by natural selection. Biology and Philosophy, 20: 673-696.

Caplan, A.L. (1981). Popper's philosophy. Nature, 290: 623-624.

Corfield, D., Schölkopf, B. and Vapnik, V. (2005). Popper, Falsification and the VCdimension. Technical Report No.145, Max Planck Institute for Biological Cybernetics.

Damuth, J. (1981). Population density and body size in mammals. Nature, 290:699-700.

Darwin, C. (1859, repr. 1962). The origin of species by means of Natural Selection or the Preservation of Favoured Races in the Struggle for Life. Collier Books, New York.

Dawkins, R. (1986). The blind watchmaker. Longman, London.

Elgin, M. (2006). There may be strict empirical laws in biology, after all. Biology and Philosophy, 21: 119-134.
Enquist, B.J., Brown, J.H. and West, G.B. (1998). Allometric scaling of plant energetics and population density. Nature, 395: 163-165.

Gardner, M. (2001). A skeptical look at Karl Popper. Skeptical Inquirer, 25 (4): 13-14.

Gould, S.J. (1989). Wonderful life. The Burgess Shale and the Nature of History. Penguin, London.

Mackenzie, D. (1999). New clues to why size equals destiny. Science 284:1607.

Mayr, E. (1976). Evolution and the diversity of life. Selected essays. The Belknap Press of the Harvard University Press, Cambridge, Massachusetts.

Mayr, E. (1998). This is Biology. The Belknap Press of the Harvard University Press, Cambridge, Massachusetts.

Popper, K.R. (1934). The Logic of Scientific Discovery. English edition 1959, Hutchinson, London. Reprinted 1990, Unwin Hyman Ltd. London.

Popper, K. (1963). Conjectures and Refutations. Routledge and Keagan Paul, London.

Popper, K. (1972). Objective Knowledge. An Evolutionary Approach. Oxford University Press, Oxford.

Ridley, M. (1985). The problems of Evolution. Oxford University Press, Oxford.

Stamos, D.N. (1996). Popper, Falsifiability, and Evolutionary Biology. Biology and Philosophy, 11: 161-191.

Thompson, D’Arcy W. (1968, 1917). On Growth and Form. Second edition, Cambridge University Press.

West, G.B., Brown, J.H. and Enquist, B.J.(1997). A general model for the origin of allometric scaling laws in biology. Science 276: 122-126.

West, G.B., Brown, J.H. and Enquist, B.J.(1999). The fourth dimension of life: fractal geometry and allometric scaling of organisms. Science 284: 1677-1679.

Williams, V.(1997). Fractal geometry gets the measure of life's scales. Science 276:34. 